\title{
Hinteres Kreuzband und posterolaterale Ecke
}

\author{
Achim Preiss, Maximilian Heitmann, Jörg Hoetzel, Karl-Heinz Frosch
}

\section{Zusammenfassung}

Verletzungen des hinteren Kreuzbands können isoliert oder in Kombination mit anderen Bandverletzungen des Kniegelenks auftreten. Ursache ist meist ein direktes Anpralltrauma des ventralen Unterschenkels oder ein Sturz auf das gebeugte Knie. Isolierte hintere Kreuzbandrupturen können in der Regel konservativ mittels einer speziellen Orthese behandelt werden. Bei der hinteren Kreuzbandverletzung ist jedoch meist die posterolaterale Ecke zusätzlich verletzt. Solche kombinierten Bandverletzungen sollten einer operativen Therapie zugeführt werden. Hierbei erfolgt die arthroskopische Rekonstruktion des hinteren Kreuzbands sowie die offene oder minimalinvasive Rekonstruktion oder Augmentation des Seitenband- und Popliteuskomplexes. Die Nachbehandlung der operativ versorgten posterolateralen Instabilität sollte mittels PCLOrthese erfolgen. Bewegungsübungen erfolgen in den ersten 6 Wochen nur in Bauchlage unter Quadrizepsanspannung. Mit der arthroskopischen hinteren Kreuzbandplastik und der posterolateralen Rekonstruktion können gute klinische Ergebnisse bei geringen Komplikationsraten erzielt werden.

\section{Anatomie und Biomechanik}

Während der embryonalen Entwicklung wandern die Kreuzbänder sekundär von dorsal in das Kniegelenk hinein. Sie liegen danach intraartikulär, jedoch extrasynovial. Das hintere Kreuzband (HKB) entspringt femoral im Bereich der medialen interkondylären Notch nahe an

OP-JOURNAL 2013; 29: 221-227

(c) Georg Thieme Verlag KG Stuttgart · New York DOI http://dx.doi.org/10.1055/s-0033-1360153

\section{Arthroscopic Joint Reconstruction - Posterior Cruciate Ligament and Posterolateral Corner}

Injuries of the posterior cruciate ligament (PCL) occur as isolated or as combined knee ligament injuries. The main cause is a direct blunt trauma to the proximal tibia or a fall on the flexed knee. Isolated injuries show good clinical results after conservative treatment, while combined injuries, affecting the posterolateral corner additionally, should be operatively. treated Arthroscopic reconstruction of the PCL followed by an open or minimally invasive reconstruction or augmentation of the peripheral structures should be performed. Aftercare comprises 6 weeks of wearing a PCL brace and movement of the joint in the prone position with active quadriceps tension. Isolated bony avulsions of the posterior cruciate ligament should also be refixated to avoid dislocation and to gain early mobilisation. In such cases we suggest either arthroscopic or mini-open surgery. With arthroscopic PCL and posterolateral reconstruction good results with a low complication rate can be achieved. richteten Translation insbesondere in Beugung dar.

Die posterolaterale Stabilität des Kniegelenks wird durch ein komplexes Zusammenspiel verschiedener anatomischer Strukturen gewährleistet. Als die wichtigsten posterolateralen Stabilisatoren werden hierbei das Lig. collaterale laterale und die Sehne des M. popliteus angesehen.

Letztere wirkt v.a. der Außenrotation des Kniegelenks entgegen, während das laterale Kollateralband den Hauptstabilisator gegen Varusstress darstellt [5]. Daneben zählen das Lig. popliteum obliquum, das Lig. popliteum arcuatum, das Lig. popliteofibulare, der Tractus iliotibialis, die Sehne des M. biceps femoris sowie das Caput laterale des M. gastrocnemius zu den Stabilisatoren der posterolateralen Ecke des Kniegelenks.

Epidemiologie und Unfallmechanismus

Verletzungen des Kniegelenks gehen in 3-37\% der Fälle mit Läsionen des hinteren Kreuzbands (HKB) einher [16]. Verkehrsunfälle und Sportunfälle spielen hierbei mit jeweils ca. $40 \%$ eine wesentliche Rolle. Schulz et al. zeigten, dass $28 \%$ aller Verletzungen des hinteren Kreuzbands auf Motorradunfälle und 25\% auf Verletzungen beim Fußball zurückzuführen sind [19].

der Knorpel-Knochen-Grenze. Die tibiale Insertion befindet sich dorsal an der proximalen Tibia ca. $1 \mathrm{~cm}$ unterhalb des Tibiaplateaus. Funktionell und anatomisch werden entsprechend dem vorderen Kreuzband 2 Bündel unterschieden. Das kräftigere anterolaterale (AL) Bündel ist in Flexion des Kniegelenks angespannt, während das schwächer ausgeprägte posteromediale (PM) Bündel seine maximale Spannung in Extension des Kniegelenks aufweist. Das hintere Kreuzband stellt den Hauptstabilisator des Kniegelenks gegenüber einer nach posterior ge-
Die häufigste Ursache stellt das direkte Anpralltrauma am ventralen proximalen Unterschenkel (dashboard injury) dar.

Aber auch ein Sturz auf das gebeugte Knie kann zur Ruptur des hinteren Kreuzbands führen. In Beugestellung sind die sekundären Stabilisatoren des Kniegelenks entspannt, sodass die Krafteinleitung isoliert auf das hintere Kreuzband erfolgt. Seltener kommt es im Rahmen von Hyperextensions- oder Rotationstraumata zu Verletzungen des hinteren Kreuzbands. 
Tab. 1 Klassifikation der HKB-Läsion nach Ausmaß der hinteren Schublade.

\begin{tabular}{ll} 
Grad 1 & $5-8 \mathrm{~mm}$ \\
\hline Grad 2 & $9-12 \mathrm{~mm}$ \\
\hline Grad 3 & $>12 \mathrm{~mm}$
\end{tabular}

In $18 \%$ aller Femurschaftfrakturen liegt eine begleitende Ruptur des hinteren Kreuzbands vor [28].

Etwa $70-80 \%$ aller operationspflichtigen HKB-Verletzungen gehen mit Läsionen der posterolateralen Ecke einher [22,29]. Die posterolateralen Strukturen tragen entscheidend zur Stabilisierung des Kniegelenks gegen posteriore Translation und Außenrotation bei $[13,23]$. Eine Verletzung derselbigen resultiert in einer posterolateralen Rotationsinstabilität.

Läsionen der posterolateralen Ecke werden jedoch häufig übersehen und im Durchschnitt erst 12 bis 30 Monate nach dem Unfall diagnostiziert [18]

\section{Anamnese und Diagnostik}

Die anamnestisch wichtigen Faktoren wie Verkehrs- oder Freizeitunfälle sind in jedem Falle zu erfragen. Patienten mit hinterer Kreuzbandruptur präsentieren häufig unspezifische Beschwerden. Oftmals haben mehrfach vorherige Arztbesuche ohne korrekte Diagnosestellung stattgefunden. Klagen Patienten über Schmerzen, so werden diese v. a. medialseitig und retropatellar angegeben. Subjektive Instabilität wird im Gegensatz zur vorderen Kreuzbandruptur seltener
Tab. 2 Klassifikation der HKB-Insuffizienz nach Harner.

\begin{tabular}{lllll} 
& A & B & C & D \\
\hline hintere Schublade in $\mathrm{mm}$ & $<5$ & $5-10$ & $11-15$ & $>15$ \\
\hline $\begin{array}{l}\text { hintere Schublade in } \\
\text { Innenrotation }\end{array}$ & abnehmend & $\begin{array}{l}\text { konstant oder } \\
\text { abnehmend }\end{array}$ & $\begin{array}{l}\text { konstant oder } \\
\text { zunehmend }\end{array}$ & zunehmend \\
\hline Varus-Instabilität & - & - & \pm & + \\
\hline Diagnose & isoliert & isoliert & kombiniert & kombiniert \\
\hline Therapieempfehlung & konservativ & $\begin{array}{l}\text { operativ, wenn } \\
\text { symptomatisch }\end{array}$ & operativ & operativ
\end{tabular}

angegeben, da eine isolierte HKB-Ruptur zur Instabilität in hohen Beugegraden führt, die nicht Teil des normalen Gangbilds sind. Ein Gelenkerguss ist aufgrund der extrasynovialen Lage nicht obligatorisch. Bei akuter Ruptur kann jedoch ein popliteales Hämatom vorliegen.

Ein wichtiges Indiz bei akuter Verletzung stellt eine prätibiale Kontusionsmarke dar.

\section{Klinische Tests zur Diagnosesicherung der posterioren Instabilität}

Spontane hintere Schublade

Hierbei liegt der Patient auf dem Rücken mit $90^{\circ}$ gebeugten Kniegelenken und aufgesetzten Füßen. Im Seitenvergleich zeigt sich die spontane hintere Schublade (Abb. 1).

\section{Hinterer Schubladen-Test}

Hierbei liegt der Patient auf dem Rücken mit $90^{\circ}$ gebeugten Kniegelenken und aufgesetzten Füßen. Die hintere Schublade wird durch manuelle Translation an der proximalen Tibia ausgelöst (Abb. 2). Bei Translation in Innen- oder Außenrotation können kombinierte Instabilitäten diagnostiziert werden.

\section{Godfrey-Test}

Hierbei befindet sich der Patient in Rückenlage. Hüft- und Kniegelenke sind $90^{\circ}$ flektiert. In dieser Position zeigt sich bei HKB-Insuffizienz eine dorsale Translation des Tibiakopfs.

\section{Aktiver Quadrizeps-Test}

Rückenlage mit $90^{\circ}$ flektiertem Knie. Eine spontane hintere Schublade reponiert sich durch Anspannung der Quadrizepsmuskulatur.

\section{Tibial Step-off Zeichen}

Bei intaktem hinteren Kreuzband ist, bei $90^{\circ}$ flektiertem Kniegelenk, das mediale Tibiaplateau ca. $10 \mathrm{~mm}$ ventral des medialen Femurkondylus zu palpieren. Bei HKB-Ruptur fehlt dieser palpable Vorsprung.

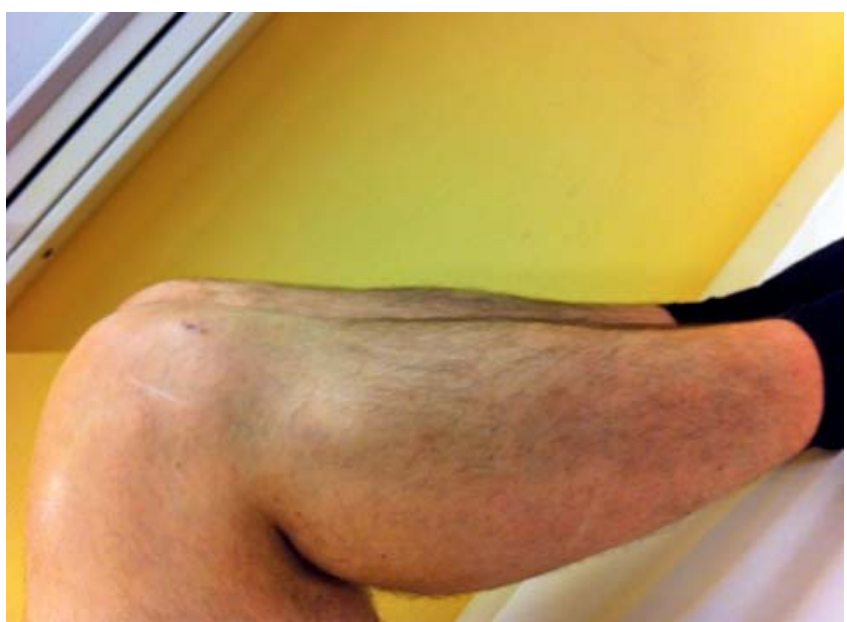

Abb. 1 Spontane hintere Schublade rechts im Seitenvergleich.

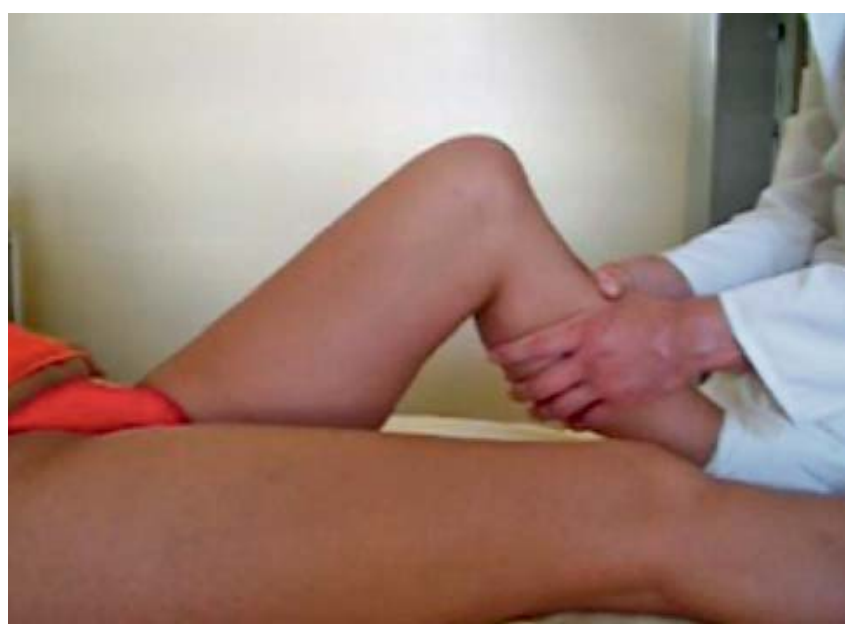

Abb. 2 Positiver hinterer Schubladentest. 


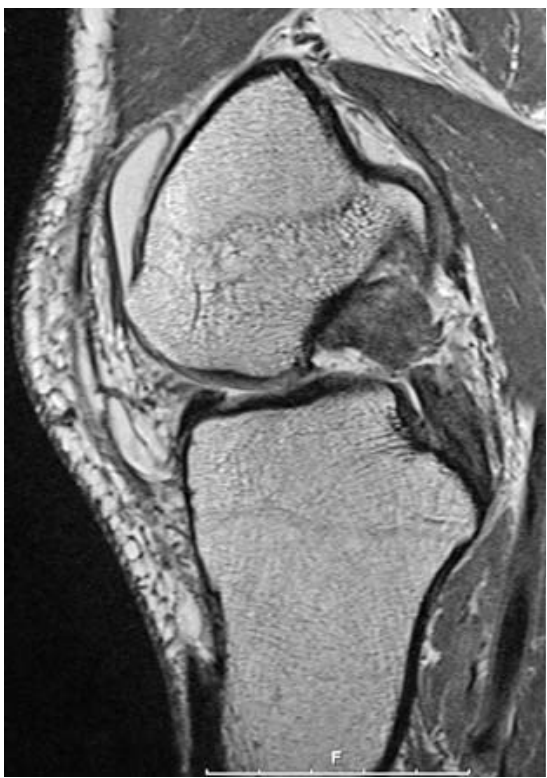

Abb. 3 MR-tomografische Darstellung einer akuten hinteren Kreuzbandruptur.

\section{Dial Test}

In Bauchlage mit $90^{\circ}$ flektierten Kniegelenken wird die Außenrotation des Unterschenkels im Seitenvergleich untersucht. Eine vermehrte Außenrotation deutet auf eine Schädigung der posterolateralen Stabilisatoren hin.

\section{Apparative Bildgebung zur Diagnostik der posterolateralen Instabilität}

Röntgenaufnahmen des Kniegelenks in 2 Ebenen stellen die Basisdiagnostik dar, um knöcherne Ausrisse erkennen zu können.

Liegt klinisch der Verdacht auf eine Achsabweichung vor, sollten Ganzbeinstandaufnahmen unter Belastung erfolgen.

Bei akuter Ruptur des hinteren Kreuzbands zeigt die Magnetresonanztomografie eine hohe Sensitivität von nahezu $100 \%$ (Abb. 3). Sie liefert zudem wichtige Hinweise auf häufige Begleitverletzungen (Menisken, Knorpel, Kollateralbänder). Bei chronischen Instabilitäten liegt die Sicherheit, eine HKB-Ruptur mittels MRT zu erfassen, hingegen nur noch bei $57 \%[20]$.

Bei chronischen Instabilitäten haben sich deshalb gehaltene Aufnahmen mittels Telos-Gerät bewährt (Abb. 4a und b).

Dabei werden gehaltene seitliche Röntgenaufnahmen des Kniegelenks in $90^{\circ}$

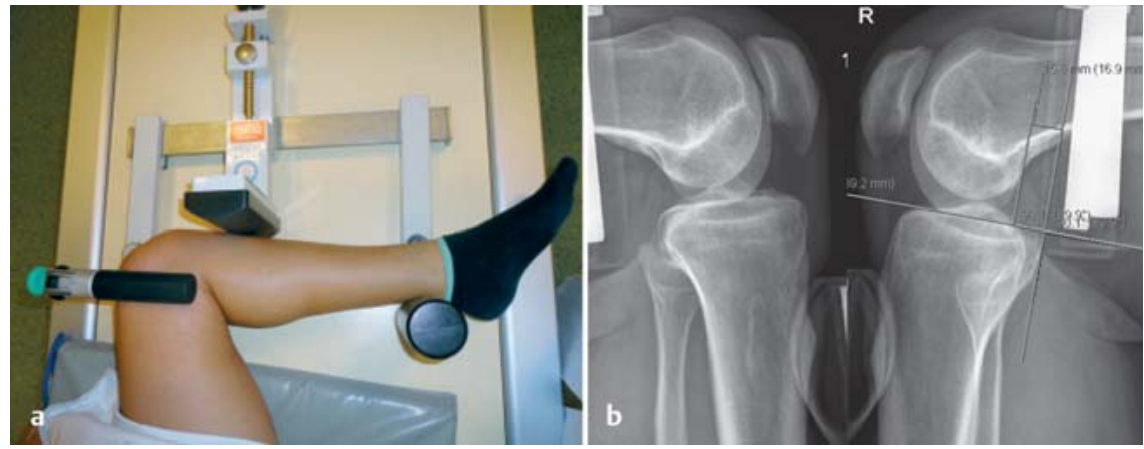

Abb. 4a und b Gehaltene Aufnahme im Telos Gerät mit Belastung des HKB (a). Die gehaltenen Aufnahmen sollten jeweils im Seitenvergleich durchgeführt werden. (b) Hintere Schublade von $-11 \mathrm{~mm}$ Seitendifferenz. Es handelt sich um eine kombinierte HKB-Ruptur mit posterolateraler Rotationsinstabilität. Eine operative Versorgung ist indiziert.

Flexion durchgeführt, während auf den Tibiakopf ein definierter Druck nach dorsal ausgeübt wird. Die Aufnahmen müssen im Seitenvergleich durchgeführt werden. Anschließend kann mittels Messmethode nach Jacobsen das Ausmaß der hinteren Schublade quantifiziert und klassifiziert werden (Tab. 1).

Insbesondere bei einer dorsalen Translation von $12 \mathrm{~mm}$ oder mehr ist von einer kombinierten HKB-Verletzung auszugehen [18]. Dabei handelt es sich meist um posterolaterale, seltener auch um posteromediale Rotationsinstabilitäten.

Bezüglich der Entscheidung, ob eine operative oder konservative Therapie erfolgen sollte, ist von entscheidender Bedeutung, ob eine isolierte HKB-Verletzung oder eine kombinierte Verletzung vorliegt. Harner entwickelte 1998 eine Klassifikation, die 4 Typen unterscheidet und eine Therapieempfehlung beinhaltet (Tab. 2).

\section{Therapie}

\section{Konservative Therapie}

Isolierte akute HKB-Rupturen können häufig primär konservativ therapiert werden.

Hierzu ist eine spezielle Orthesenbehandlung notwendig. Wir präferieren eine flexible PCL-Orthese mit einstellbarem Bewegungsausmaß (Jack PCL, Fa. Albrecht). Diese sollte für insgesamt 3 Monate getragen werden. Alternativ kann die ersten 6 Wochen eine starre Orthese mit Pelotte im Bereich der dorsalen proximalen Tibia getragen werden (PTSOrthese). In den ersten 6 Wochen erfolgt die Teilbelastung unter $20 \mathrm{~kg}$ an Unterarmgehstützen. Begleitend sollten iso- metrische Quadrizepsanspannungen sowie physiotherapeutische Übungsbehandlungen in Bauchlage bis $90^{\circ}$ Flexion erfolgen, um eine nach dorsal gerichtete Translation des Tibiakopfs zu vermeiden. In den meisten Fällen lassen sich damit gute und sehr gute klinische Ergebnisse erzielen [21]. Sind im MRT jedoch eine deutliche Dislokation oder Dehiszenz der Bandstümpfe des HKB zu finden, ist auch primär die operative Therapie zu erwägen.

\section{Operative Therapie}

\section{Arthroskopischer hinterer Kreuzbandersatz}

Der arthroskopische hintere Kreuzbandersatz ist technisch anspruchsvoll. Er erfordert Erfahrung in der arthroskopischen Chirurgie, gute anatomische Kenntnisse und Orientierung, welche intraoperativ visuell umgesetzt werden müssen.

\section{Transplantatwahl}

Zur Rekonstruktion des hinteren Kreuzbands stehen verschiedene autogene Transplantate zur Verfügung.

Die Patellarsehne (BPTB: bone-patellar tendon-bone) bietet mit den beiden endständigen Knochenblöcken den Vorteil der schnellen knöchernen Einheilung [27]. Bezüglich der Sehnenentnahme liegen langfristige Erfahrungen aus der vorderen Kreuzbandchirurgie vor. Nachteilig ist die Entnahmemorbidität im Sinne von Schmerzen und Sensibilitätsstörungen [1]. Zudem wird der Streckapparat geschwächt, der muskulär der posterioren Translation der Tibia entgegenwirkt. Operationstechnisch kann das BPTB-Transplantat beim Einziehen durch den starken Richtungswechsel am dorsalen Tibiakopf Probleme bereiten. Auch 


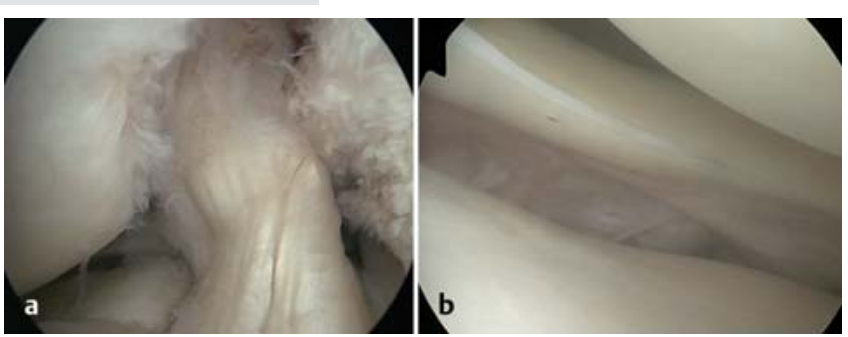

Abb. $5 \mathrm{a}$ und $\mathrm{b}$ a Lockeres vorderes Kreuzband („floppy ACL") als Zeichen der hinteren Kreuzbandruptur. b Weites laterales Kompartiment bei kombinierter Instabilität.

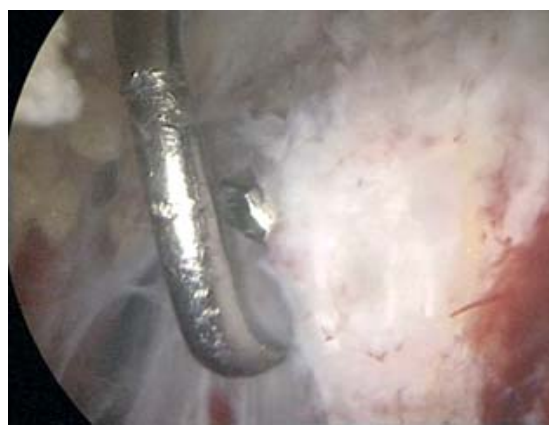

Abb. 6 Platzierung des tibialen Zielgeräts und des Ösenbohrdrahts im Zentrum des tibialen HKB-Stumpfes.

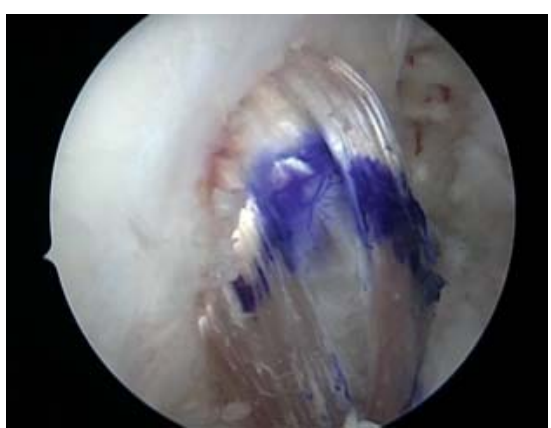

Abb. 7 Dargestellt ist ein HKB-Transplantat, welches femoral bei 11 Uhr direkt an der Knorpel-Knochen-Grenze zu liegen kommt.

die Transplantatlänge kann ein limitierender Faktor sein, sodass das BPTBTransplantat nicht regelhaft für den HKB-Ersatz empfohlen wird.

Die Quadrizepssehne bietet mit dem Knochenblock aus der Patella einseitig den Vorteil der knöchernen Einheilung, auf der gegenüberliegenden Seite muss eine tendoossäre Integration stattfinden [6].

Die beiden o.g. autogenen Transplantate finden bei uns in der Revisionschirurgie ihre Anwendung. Primäre Transplantatwahl stellen jedoch die Hamstringsehnen (Semitendinosus und Gracilis) dar.

Das Transplantat sollte für die HKB-Rekonstruktion eine Länge von ca. $11 \mathrm{~cm}$ besitzen. Hierzu werden die beiden Seh-

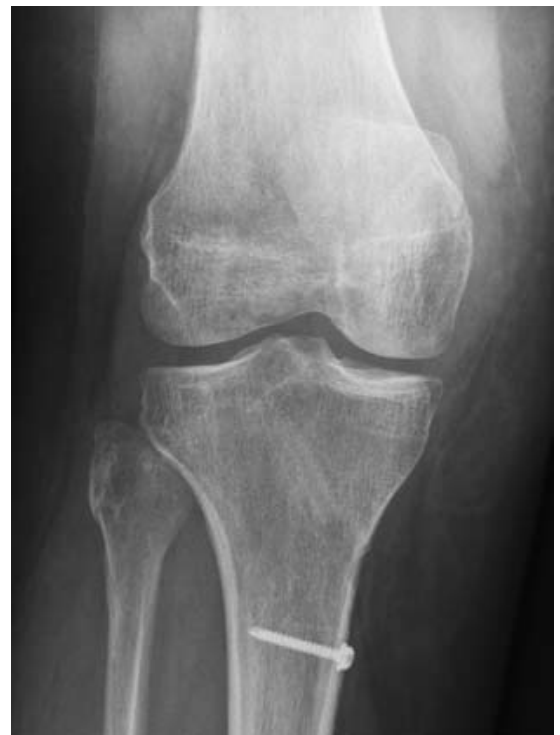

Abb. 8 Postoperatives Röntgenbild nach arthroskopischer HKB-Rekonstruktion (zusätzliche Kleinfragmentschraube tibial als Backup).

nen 4-fach gelegt. Femoralseitig wird ein Durchzugsfaden ohne Vernähen des Transplantats verwendet. Tibial werden die Sehnen auf einer Strecke von $3 \mathrm{~cm}$ mittels nicht resorbierbarem Faden (FibreWire2, Fa. Arthrex) vernäht.

\section{Single oder Double Bundle?}

Derzeit liegt keine Evidenz vor, dass eine Rekonstruktion in Zweibündeltechnik der Einzelbündel-Rekonstruktion im klinischen Outcome überlegen ist [8].

Einige Studien zeigen zwar bessere Werte bei den objektiven biomechanischen Daten, dies spiegelt sich jedoch nicht in den subjektiven Scores wider [26].

Bei kombinierter Rekonstruktion von HKB und posterolateraler Ecke weist die Doppelbündel-Rekonstruktion keinen Vorteil auf [2].

Wir führen die Rekonstruktion standardmäßig in „single bundle“-Technik durch. Hierbei wird der femorale Bohrkanal im Footprint des AL-Bündels platziert.

\section{Operationstechnik}

Die Lagerung des zu operierenden Beines erfolgt in einem elektrisch verstellbaren Beinhalter, um verschiedene Flexionsgrade während der Operation einstellen zu können. Bei gleichzeitiger vorderer Kreuzbandersatzplastik oder Rekonstruktion und Augmentation peripherer Bandstrukturen wird das kontralaterale Bein zur Sehnenentnahme ebenfalls chirurgisch desinfiziert und steril abgedeckt. Zu Beginn steht die Stabilitätsprüfung des Kniegelenks in Narkose, um die Diagnose zu verifizieren und begleitende Instabilitäten zu eruieren. Anschließend erfolgt die diagnostische Arthroskopie, um Begleitverletzungen wie Meniskusläsionen oder Knorpelschäden zu diagnostizieren und zu adressieren.

Bei hinterer Kreuzbandruptur liegt häufig aufgrund der hinteren Schublade ein sog. „floppy ACL“ vor. Dies darf nicht als vordere Kreuzbandverletzung fehlgedeutet werden (Abb. 5 a).

Eine laterale Instabilität kann arthroskopisch gut anhand eines weiten lateralen Kompartiments erkannt werden (Abb.5b).

Nun erfolgt die Anlage eines posteromedialen Portals, über welches der tibiale HKB-Stumpf präpariert und dargestellt wird. Als wichtige Orientierung dient hier die dorsale Tibiakante. Anschließend erfolgt die Visualisierung von posteromedial. Hiernach wird über das anteromediale Portal ein Zielgerät im Bereich des distalen lateralen HKB-Ansatzes platziert. Über dieses Zielgerät wird ein Ösendraht gebohrt (Abb.6) und dieser anschließend mittels Bohrer der zuvor ausgemessenen Transplantatstärke überbohrt. Hiernach wird für den femoralen Bohrkanal über das anterolaterale Portal ein Bohrdraht im Footprint des AL-Bündels ca. $5 \mathrm{~mm}$ von der KnorpelKnochen-Grenze eingebracht. Auch hier erfolgt wieder das Überbohren in der zuvor gemessenen Stärke des Transplantats. Über Shuttle-Fäden wird nun das Transplantat über den tibialen Bohrkanal nach intraartikulär und dann nach femoral eingezogen (Abb. 7). Die Fixation führen wir mit Bio-Interferenzschrauben (Milagro, Fa. Depuy Synthes) sowie tibial mit einer zusätzlichen Backup-Kleinfragmentschraube durch (Abb. 8). Im Revisionsfall oder bei ausgesprochen weichem Knochen haben sich Titan-Interferenzschrauben bewährt. 


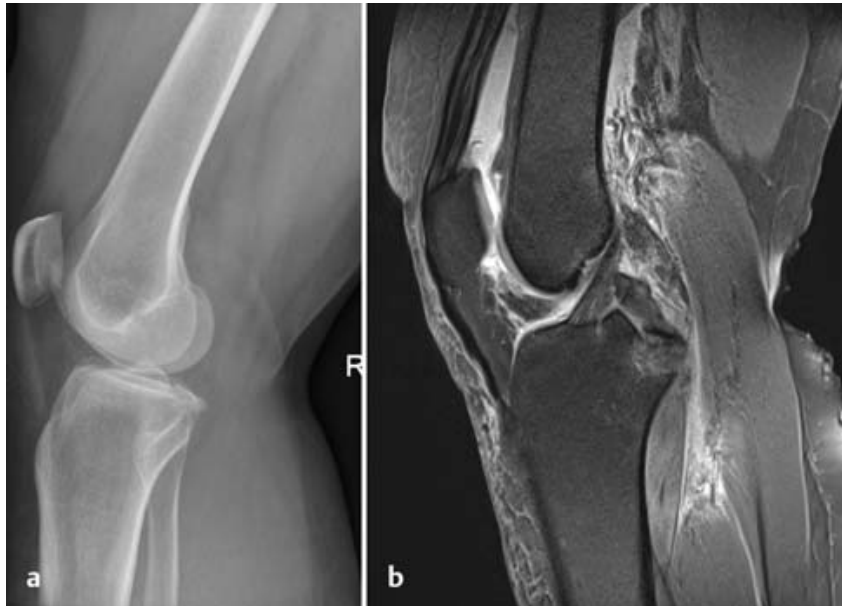

Abb. 9a und b Akuter knöcherner Ausriss des hinteren Kreuzbands im Röntgen (a) und MRT (b).

Bei isolierter hinterer Instabilität können nach Rekonstruktion mittels HamstringSehnentransplantat in bis zu $90 \%$ gute klinische Ergebnisse erzielt werden [4, $7,9,25]$.

\section{Knöcherne HKB-Ausrisse}

Knöcherne Ausrisse des hinteren Kreuzbands (Abb.9a und b) sollten frühzeitig refixiert werden. Hierdurch kann auf eine mehrere Wochen lange Immobilisation des Kniegelenks in einer Orthese verzichtet werden.

Die Refixation kann arthroskopisch mittels einer „Zugfadenosteosynthese“ erfolgen. Dabei wird das Fragment arthroskopisch angeschlungen und reponiert, der Faden tibial transossär nach ventral herausgezogen und dort mittels Schraube, Suture Washer oder Endobutton fixiert. Das Fadenmaterial sollte eine hohe Reißfestigkeit aufweisen und nicht oder wenig resorbierbar sein.

Alternativ können knöcherne HKB-Ausrisse auch direkt über den von Frosch et al. beschriebenen minimalinvasiven posteromedialen Zugang mittels kanülierter Schrauben oder bei mehrfragmentären Ausrissen mittels Fadenanker refixiert werden [11]. Diese Methode stellt für den arthroskopisch weniger versierten Operateur eine schnelle und sichere Alternative dar (Abb. 10 a und b).

\section{Kombinierte Verletzungen des hinteren Kreuzbands und der posterolateralen Ecke}

Bei HKB-Instabilitäten mit mehr als $12 \mathrm{~mm}$ dorsaler Schublade in den gehaltenen seitlichen Aufnahmen handelt es

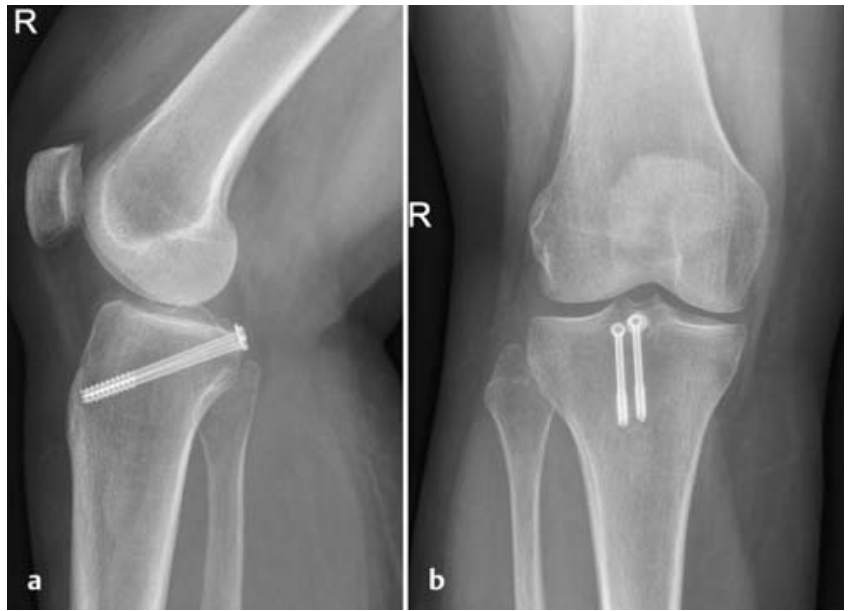

Abb. 10a und b Direkte Verschraubung eines knöchernen Ausrisses des hinteren Kreuzbands.
Eine isolierte HKB-Rekonstruktion vermag bei kombinierten Instabilitäten lediglich die hintere Schublade zu verringern, nicht aber die rotatorische Instabilitätskomponente [3].

Isolierte Rekonstruktionen des hinteren Kreuzbands sind bei kombinierter Instabilität erhöhtem biomechanischen Stress ausgesetzt und somit anfällig für ein Transplantatversagen [15].
Abb. 11 Offene posterolaterale Stabilisierung nach Larson mittels autologer Semitendinosussehne im Rahmen einer Knieluxation. Zuvor wurden sämtliche rupturierten Bänder genäht und refixiert.

Die operative Versorgung des HKB erfolgt wie oben beschrieben in arthroskopischer Technik. Um die posterolaterale Rotationsinstabilität zu addressieren, sind eine Vielzahl von Rekonstruktionsmöglichkeiten entwickelt worden. Am häufigsten wird die Rekonstruktion nach Larson angewendet, die einen anatomischen Kompromiss zur Rekonstruktion der posterolateralen Strukturen darstellt. Als Transplantat dient hierbei die kontralaterale Semitendinosus- oder Gracilissehne, die triangelförmig durch einen Bohrkanal im Fibulaköpfchen gezogen und femoral mittels Interferenzschraube verankert wird.

Abb. 11 zeigt den Fall einer akuten Verletzung der posterolateralen Ecke mit primärer Naht des Kollateralbandkomplexes und zusätzlicher Augmentation nach Larson mittels autologer Semitendinosussehne.

Die posterolaterale Augmentation nach Larson kann auch in minimalinvasiver 


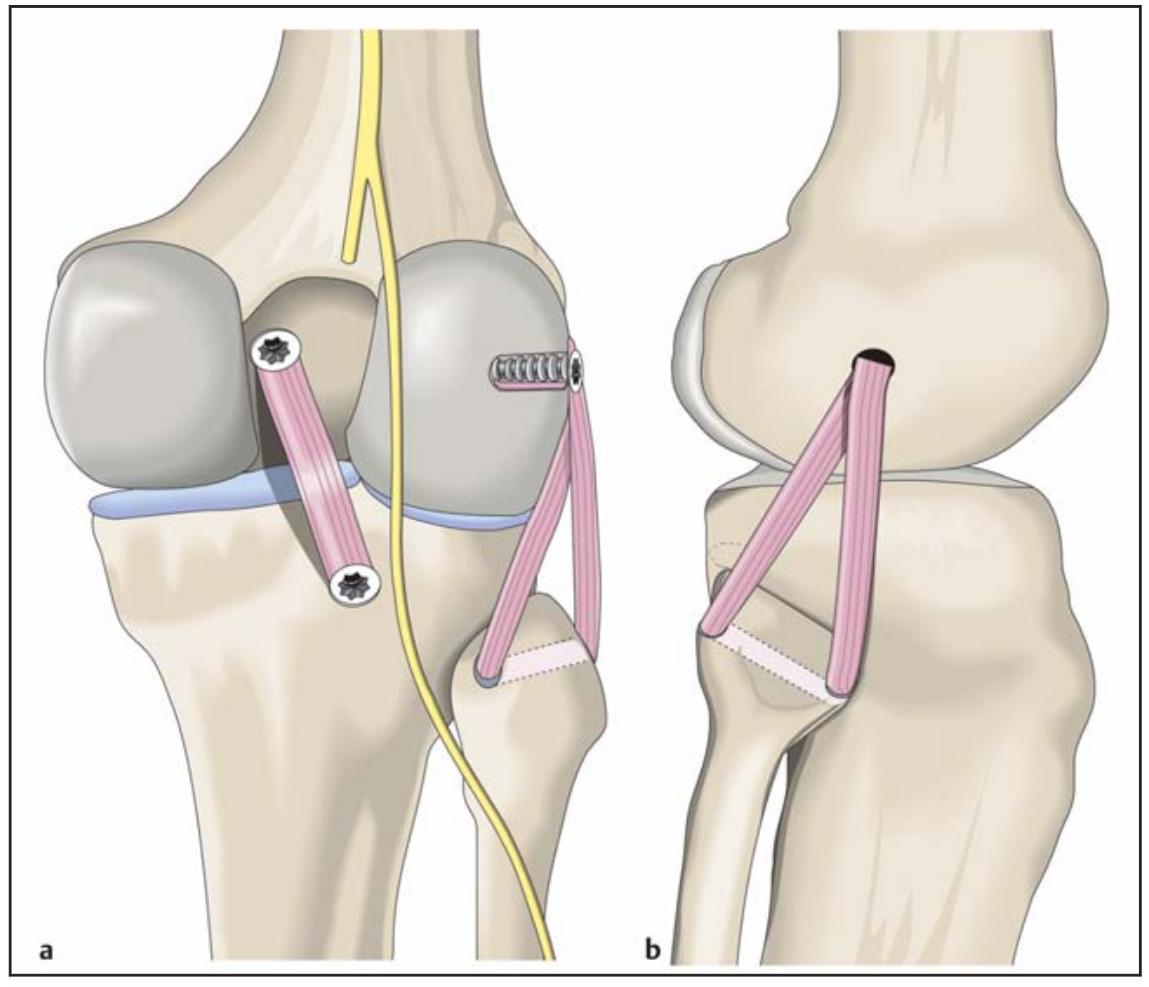

Abb. 12a und b Schemazeichnung der minimal invasiven, jedoch extraanatomischen Rekonstruktion der posterolateralen Ecke nach Larson. Femoral wird nur 1 Tunnel verwendet, distal wird ein Kanal in die Fibulaspitze gebohrt, durch den das Transplantat hindurchgezogen wird. Mit der Larson-Technik, welche sehr einfach und Zeit sparend durchgeführt werden kann, wird die dorsale Instabilität um nur ca. 50\% reduziert.

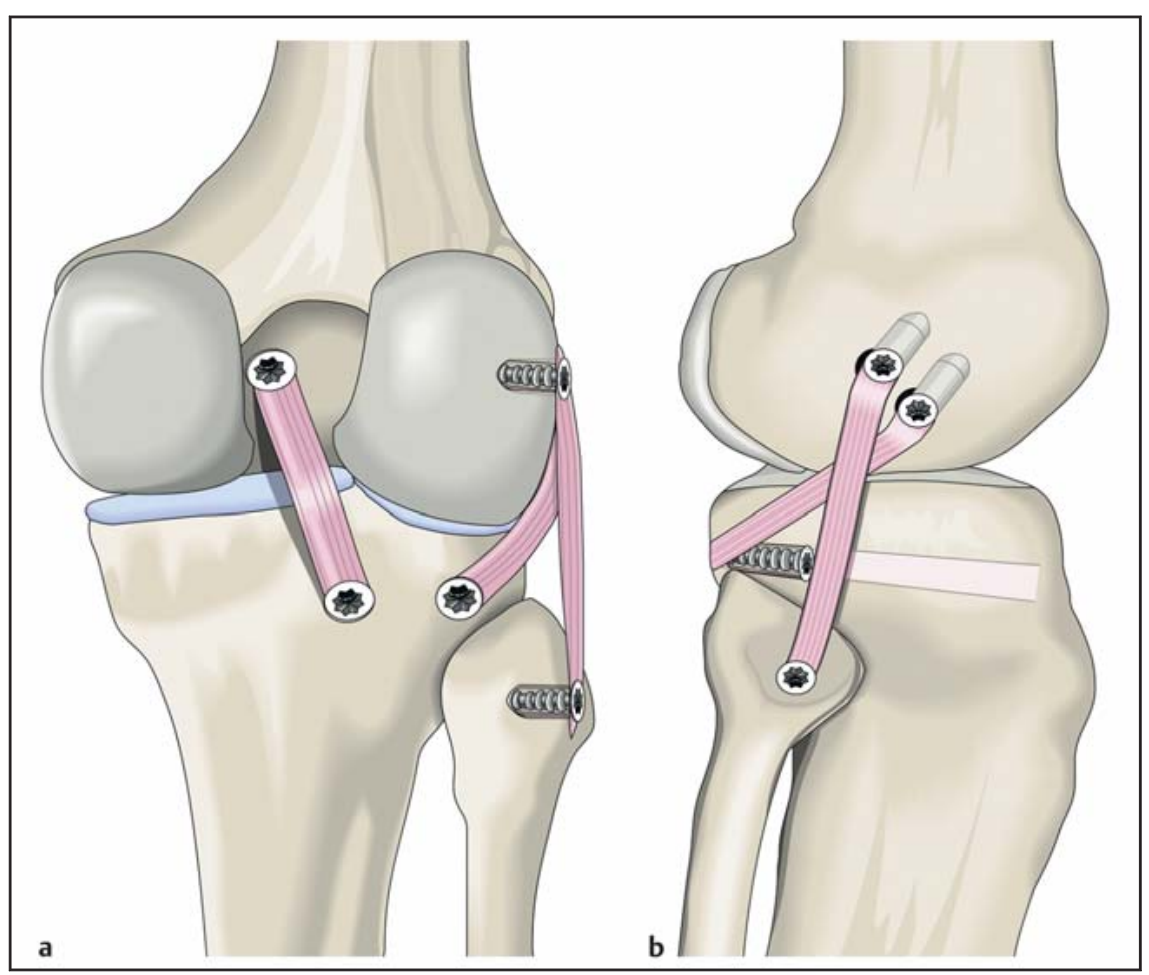

Abb. 13a und b Die anatomische Rekonstruktion der posterolateralen Ecke im Rahmen der kombinierten Versorgung. Insbesondere die Rekonstruktion des Popliteuskomplexes ist anspruchsvoll, da ein tibialer Kanal im Bereich der schwer zugänglichen, posterolateralen Ecke angelegt werden muss. Dies kann offen oder arthroskopisch erfolgen. Femoral werden bei der anatomischen Rekonstruktion der posterolateralen Ecke 2 Kanäle im Abstand von durchschnittlich $12 \mathrm{~mm}$ gebohrt. Die postoperative Stabilität ist mit den anatomischen Techniken in der Regel deutlich höher als mit der Larson-Technik.
Technik durchgeführt werden. Dabei ist jedoch zu beachten, dass der N. peroneus in unmittelbarer Nähe zur augmentierten Sehne $\mathrm{zu}$ finden ist und geschont werden muss (Abb. 12a).

Mittels kombinierter Rekonstruktion von HKB und posterolateraler Ecke lassen sich signifikant bessere klinische Ergebnisse erzielen als mittels isolierter HKB-Rekonstruktion [10]. Insgesamt lassen sich jedoch mit älteren Techniken nur bis zu $64 \%$ sehr gute und gute klinische Ergebnisse bei Kombinationsverletzungen erreichen [24]. Neuere Techniken der Popliteusrekonstruktion mit tibialem Tunnel zeigen deutlich bessere Ergebnisse [17]. Dabei wird der Popliteuskomplex möglichst anatomisch rekonstruiert, zusätzlich das laterale Kollateralband (Abb. 12 b). Bei der aufwendigen offenen Rekonstruktion mit hohem Transplantatverbrauch werden exzellente klinische Ergebnisse beschrieben. Die radiologisch gemessene dorsale Translation postoperativ betrug dabei im Schnitt 1,2 mm [12]. Jung et al. konnten zeigen, dass die technisch einfachere Stabilisierung der posterolateralen Ecke mittels fibularem distalen Tunnel gleich gute klinische Ergebnisse liefert wie die Technik mittels tibialem Tunnel. $\mathrm{Zu}$ beachten ist dabei jedoch, dass die mittlere dorsale Instabilität präoperativ nur $10 \mathrm{~mm}$ war, sodass also mit großer Wahrscheinlichkeit auch isolierte dorsale Instabilitäten mit eingeschlossen wurden [14].

Der femorale Kanal sollte 5-7 mm distal und anterior des lateralen Epicondylus femoris eingebracht werden [14] (Abb. 13a und b).

Prognostisch wichtiger Faktor bei der Rekonstruktion ist u.a. auch die möglichst kurze Zeitspanne zwischen Verletzung und Rekonstruktion [24].

\section{Fazit}

Akute hintere Kreuzbandrupturen mit dorsaler Schublade bis $10 \mathrm{~mm}$ können mit guten klinischen Ergebnissen konservativ mittels Orthesenbehandlung therapiert werden. Bei stärker ausgeprägter dorsaler Schublade liegt jedoch meist eine Kombinationsverletzung des hinteren Kreuzbands und der posterolateralen Stabilisatoren des Kniegelenks mit daraus resultierender posterolateraler Rotationsinstabilität vor, die eine operative Therapie erforderlich macht. Hierbei erfolgt die arthroskopische Re- 
konstruktion des hinteren Kreuzbands mittels autologem Hamstring-Sehnentransplantat. Zusätzlich erfolgt die Rekonstruktion der posterolateralen Ecke. Die Technik nach Larson stellt hierbei eine häufig angewendete Technik dar. Neuere anatomische Rekonstruktionsverfahren scheinen funktionell jedoch überlegen. Isolierte knöcherne Ausrisse des HKB sollten arthroskopisch oder minimalinvasiv refixiert werden.

\section{Literatur}

${ }^{1}$ Aglietti P, Giron F, Buzzi R et al. Anterior cruciate ligament reconstruction: bone-patellar tendon-bone compared with double semitendinosus and gracilis tendon grafts. A prospective, randomized clinical trial. J Bone Joint Surg [Am] 2004; 86: 2143-2155

2 Apsingi S, Nguyen T, Bull AM et al. Control of laxity in knees with combined posterior cruciate ligament and posterolateral corner deficiency: comparison of single-bundle versus double-bundle posterior cruciate ligament reconstruction combined with modified Larson posterolateral corner reconstruction. Am J Sports Med 2008; 36: 487-494

3 Apsingi S, Nguyen T, Bull AM et al. The role of PCL reconstruction in knees with combined PCL and posterolateral corner deficiency. Knee Surg Sports Traumatol Arthrosc 2008; 16: $104-111$

${ }^{4}$ Chan YS, Yang SC, Chang CH et al. Arthroscopic reconstruction of the posterior cruciate ligament with use of a quadruple hamstring tendon graft with 3- to 5-year follow-up. Arthroscopy 2006; 22: 762-770

${ }^{5}$ Chen $\mathrm{CH}$. Surgical treatment of posterior cruciate ligament injury. Chang Gung Med 2007; 30: 480-492

${ }^{6}$ Chen $\mathrm{CH}$, Chen WJ, Shih CH et al. Arthroscopic posterior cruciate ligament reconstruction with quadriceps tendon autograft: minimal 3 years follow-up. Am J Sports Med 2004 32: $361-368$

${ }^{7}$ Chen CH, Chuang TY, Wang KC et al. Arthroscopic posterior cruciate ligament reconstruction with hamstring tendon autograft: results with a minimum 4-year follow-up. Knee Surg Sports Traumatol Arthrosc 2006; 14: 1045-1054

${ }^{8}$ Christel P. Basic principles for surgical reconstruction of the PCL in chronic posterior knee instability. Knee Surg Sports Traumatol Arthrosc 2003; 11: 289-296
${ }^{9}$ Deehan DJ, Salmon LJ, Russell VJ et al. Endoscopic single-bundle posterior cruciate ligament reconstruction: results at minimum 2year follow-up. Arthroscopy 2003; 19: 955962

10 Freeman RT, Duri ZA, Dowd GS. Combined chronic posterior cruciate and posterolateral corner ligamentous injuries: a comparison of posterior cruciate ligament reconstruction with and without reconstruction of the posterolateral corner. Knee 2002; 9: 309-312

${ }^{11}$ Frosch $K$, Proksch N, Preiss A et al. [Treatment of bony avulsions of the posterior cruciate ligament (PCL) by a minimally invasive dorsal approach]. Oper Orthop Traumatol 2012; 24: 348-353

12 Geeslin AG, Laprade RF. Outcomes of treatment of acute grade-III isolated and combined posterolateral knee injuries: a prospective case series and surgical technique. J Bone Joint Surg [Am] 2011; 93: 1672-1683

13 Harner CD, Vogrin TM, Hoher J et al. Biomechanical analysis of a posterior cruciate ligament reconstruction. Deficiency of the posterolateral structures as a cause of graft failure. Am J Sports Med 2000; 28: 32-39

${ }^{14}$ Jung YB, Jung HJ, Kim SJ et al. Posterolateral corner reconstruction for posterolateral rotatory instability combined with posterior cruciate ligament injuries: comparison between fibular tunnel and tibial tunnel techniques. Knee Surg Sports Traumatol Arthrosc 2008; 16: 239-248

${ }^{15}$ Laprade RF, Muench C, Wentorf F et al. The effect of injury to the posterolateral structures of the knee on force in a posterior cruciate ligament graft: a biomechanical study. Am J Sports Med 2002; 30: 233-238

${ }^{16}$ Mariani PP, Becker R, Rihn J et al. Surgical treatment of posterior cruciate ligament and posterolateral corner injuries. An anatomical, biomechanical and clinical review. Knee 2003; 10: 311-324

17 Markolf KL, Graves BR, Sigward SM et al. Popliteus bypass and popliteofibular ligament reconstructions reduce posterior tibial translations and forces in a posterior cruciate ligament graft. Arthroscopy 2007; 23: 482-487

18 Pacheco RI, Ayre CA, Bollen SR. Posterolateral corner injuries of the knee: a serious injury commonly missed. J Bone Joint Surg [Br] 2011: 93: 194-197

${ }^{19}$ Schulz MS, Russe K, Weiler A et al. Epidemiology of posterior cruciate ligament injuries. Arch Orthop Trauma Surg 2003; 123: 186191

20 Servant CT, Ramos JP, Thomas NP. The accuracy of magnetic resonance imaging in diagnosing chronic posterior cruciate ligament injury. Knee 2004; 11: 265-270
${ }^{21}$ Shelbourne KD, Clark M, Gray T. Minimum 10year follow-up of patients after an acute, isolated posterior cruciate ligament injury treated nonoperatively. Am J Sports Med 2013; 41: $1526-1533$

22 Strobel MJ, Weiler A. Therapie der chronischen HKB-Läsion. Arthroskopie 2006; 19: 243-257

${ }^{23}$ Vogrin TM, Hoher J, Aroen A et al. Effects of sectioning the posterolateral structures on knee kinematics and in situ forces in the posterior cruciate ligament. Knee Surg Sports Traumatol Arthrosc 2000; 8: 93-98

${ }^{24}$ Wang CJ. Injuries to the posterior cruciate ligament and posterolateral instabilities of the knee. Chang Gung Med J 2002; 25: 288-297

${ }^{25}$ Wang CJ, Chen HS, Huang TW. Outcome of arthroscopic single bundle reconstruction for complete posterior cruciate ligament tear. Injury 2003; 34: 747-751

26 Yoon KH, Bae DK, Song SJ et al. A prospective randomized study comparing arthroscopic single-bundle and double-bundle posterior cruciate ligament reconstructions preserving remnant fibers. Am J Sports Med 2011; 39: 474-480

27 Yoshiya S, Nagano M, Kurosaka M et al. Graft healing in the bone tunnel in anterior cruciate ligament reconstruction. Clin Orthop Relat Res 2000: 278-286

${ }^{28}$ Zantop T. Begleitende Verletzungen des hinteren Kreuzbandes bei Femurschaftfrakturen. In: Joint German Congress of Orthopaedics and Trauma Surgery, Berlin, 2006

29 Zantop T, Petersen W. [Modified Larson technique for posterolateral corner reconstruction of the knee]. Oper Orthop Traumatol 2010; 22: 373-386

\section{Dr. med. Achim Preiss}

Facharzt

Maximilian Heitmann

Assistenzarzt

Jörg Hoetzel

Assistenzarzt

Prof. Dr. med. Karl-Heinz Frosch

Chefarzt

Sektion Knie- und Schulterchirurgie, Sporttraumatologie

Chirurgisch-Traumatologisches

Zentrum

Asklepios Klinik St. Georg

Lohmühlenstraße 5

20099 Hamburg

k.frosch@asklepios.com 